\title{
Management of refractory pityriasis rubra pilaris: challenges and solutions
}

This article was published in the following Dove Press journal:

Clinical, Cosmetic and Investigational Dermatology

9 November 2017

Number of times this article has been viewed

\section{Gaia Moretta \\ Erika V De Luca \\ Alessandro Di Stefani \\ Institute of Dermatology, Fondazione Policlinico Universitario A. Gemelli, Catholic University of the Sacred Heart, Rome, Italy}

\begin{abstract}
Pityriasis rubra pilaris (PRP) is a rare chronic inflammatory papulosquamous skin disease. Its clinical presentation and evolution is very variable. The most frequent clinical features are follicular papules, progressing to yellow-orange erythroderma with round small areas of normal skin and the well-demarcated palmoplantar keratoderma. Actually, six different types of PRP have been described based on clinical characteristics, age of onset, and prognosis. The pathogenesis is still unknown, and treatment can be challenging. Available treatments are mainly based on case reports or case series of clinical experience because no controlled randomized trials have never been performed because of the rarity of the condition. Traditional systemic treatment consists in retinoids, which are actually considered as first-line therapy, but refractory cases that do not respond or relapse after drug interruption do exist. In recent years, numerous reports have demonstrated the efficacy of new agents such as biological drugs. This article is an overview on available therapeutic options, in particular for refractory forms of PRP.
\end{abstract}

Keywords: pityriasis rubra pilaris, biologics, retinoids, papulosquamous skin diseases

\section{Introduction}

Pityriasis rubra pilaris (PRP) is a rare chronic papulosquamous disorder of keratinization, with an incidence between 1 in 5000 and 1 in 50000 with no gender predilection. ${ }^{1}$ The pathogenesis is still unclear: it has been hypothesized that it is induced by an abnormal immune response toward different antigenic stimuli such as infections, trauma, and malignancy. ${ }^{2,3}$ Most of the cases are sporadic, but familial forms of the disease have been described, in particular linked to mutations in the gene CARD. ${ }^{4-6}$ In PRP, the epidermis is in a hyperkinetic state with an increase in turnover of the follicular keratinocytes. It has been suggested a pathogenetic role of a deficiency or a malfunction of vitamin $\mathrm{A}^{7}$ or decreased serum level of retinol-binding protein, which is the carrier of vitamin $\mathrm{A},{ }^{8}$ along with some clinical similarities to phrynoderma (a cutaneous manifestation of vitamin A deficiency). ${ }^{9}$

\section{Clinical presentation}

Generally, PRP is characterized by small follicular papules $\sim 1 \mathrm{~mm}$ in diameter with a central keratotic plug, coalescing scaly yellow pink patches, and by palmoplantar keratoderma. Lesions are symmetrical and diffuse and appear first on the extensor surfaces of the extremities, shoulders, and buttocks, usually spreading caudally with possible development of erythroderma. ${ }^{1,7}$ Differential diagnosis with psoriatic erythroderma can be made if typical roundish areas of normal skin, the island of sparing, can be identified.
Correspondence: Alessandro Di Stefan Institute of Dermatology, Catholic University of Rome, Largo Agostino Gemelli 8, 00168 Rome, Italy

Tel +390630154227

Fax +390630154919

Email alessandro.distefani@gmail.com 
Scaling is frequent and tends to be rather fine and pityriasiform on the face and scalp and coarser on the lower half of the body. The skin on the palmoplantar regions tends to become thickened and yellow-orange in color, with well-demarcated borders. Sometimes patients complain of fever, chills, itching, and malaise. ${ }^{1,7}$ Nail involvement is also very common with subungueal hyperkeratosis and yellow-brown discolorations. ${ }^{10}$ Oral mucosa can also be involved with white spots and lines: erythematous painful lesions with white streaks can appear on the buccal mucosa, gingivae, and tongue, mimicking lichen planus' signs. ${ }^{11}$ In adults, skin lesions appear first on the face and scalp, spreading in a caudal direction, while in young patients, PRP usually stars on the lower half of the body. ${ }^{12}$ However, PRP's clinical presentation and evolution are very variable.

\section{Classification}

In 1980, PRP has been classified into the following five types by Griffiths ${ }^{13-15}$ based on clinical features, age of onset, and prognosis: classic adult type I, atypical adult type II, classic juvenile type III, circumscribed juvenile type IV, and atypical juvenile type V. Later, the VI type which is the human immunodeficiency virus (HIV)-related form, has been added. ${ }^{16}$ The classic adult type $\mathrm{I}$ is the most common with an acute onset and accounts $\sim 50 \%$ of patients. Commonly, it begins with the onset of a single erythematous patch on the upper half of the body. Successively, the cutaneous lesions spread caudally within a few weeks or months and can evolve to erythroderma with the typical islands of sparing and palmoplantar yellow-orange hyperkeratosis. The duration of this form is $\sim 3-4$ years. ${ }^{13-15}$ The atypical adult type II, developed by $5 \%$ of the patients, has a chronic course up to 20 years. It is characterized by ichthyosiform lesions, especially on the legs, in association with alopecia and areas of eczema. ${ }^{13-15}$ The classic juvenile type III is similar to adult-onset type I but affects children (10\% of patients) and has a clinical course more favorable than adults, usually going to remission after 1 year. ${ }^{12}$ The circumscribed juvenile type IV affects children and young adults $(25 \%$ of patients) and develops follicular hyperkeratosis and erythema usually only on the knees and elbows with well-demarcated borders. Palmoplantar involvement is also characteristic in this form with keratoderma, or dorsal involvement of hands and feet. It remains localized in most of the cases but can be characterized by remissions and exacerbations. ${ }^{12,17,18}$ The atypical juvenile type $\mathrm{V}$ occurs in the first few years of life in up to $5 \%$ of patients; it is chronic and characterized by follicular hyperkeratosis and scleroderma-like skin lesions on the hands and feet, whereas erythema is not prominent. ${ }^{13-15}$ The HIV-related form VI is similar to type I, with a symmetrical, pruritic eruption composed of erythematous and desquamating follicular papules, but it has a more severe course and tends to be refractory to treatment. Prominent follicular plugging with formation of spicules is another common finding in this form; however, other follicular manifestations can be associated to HIV-related PRP, such as acne conglobata, hidradenitis suppurativa, and lichen spinulosus. ${ }^{16}$ This classification is not very strict: in fact, it is not always possible to assign every form of PRP to a single type because intermediate forms and transition from one type to another can occur. ${ }^{15}$

\section{Diagnosis}

The diagnosis is primarily clinical, based on typical abovementioned findings. However, sometimes, especially when PRP is in the erythroderma state, it is rather difficult to establish a clear-cut clinical diagnosis and it can be misdiagnosed as psoriasis, follicular eczema, follicular ichthyosis, generalized hypersensitivity reaction, T-cell lymphoma, and lichen planopilaris. ${ }^{1,7}$

In the last few years, dermoscopy has been applied also to evaluate inflammatory cutaneous diseases, ${ }^{19}$ and it has been proposed as possible support in the clinical diagnosis, allowing a better distinction of PRP from psoriasis: psoriatic lesions generally show regularly distributed dotted vessels on a light red background while PRP lesions show more irregular linear and dotted vessels and round/oval yellowish areas. ${ }^{19-21}$ Recently, two cases of adult and juvenile PRP have been studied also with reflectance confocal microscopy that might represent an adjunctive tool for improving the diagnostic confidence. ${ }^{21}$ Actually, the clinicopathological correlation remains the "gold standard" for the diagnosis of PRP by excluding other dermatoses especially psoriasis and cutaneous lymphoma. Typical histopathological features of PRP, although not pathognomonic, are alternating orthokeratosis and parakeratosis in both vertical and horizontal directions forming the checkerboard pattern; other frequent findings are hypergranulosis, follicular plugging, broad rete ridges, narrow dermal papillae, and sparse superficial perivascular lymphocytic infiltrate. ${ }^{22}$

\section{Therapy}

The management of PRP involves topical and systemic therapies, according to extension and severity of disease (Table 1).

\section{Topical treatments}

Topical treatment is indicated for localized forms such as PRP type III and is always recommended also in association with systemic therapy for severe forms to reduce cutaneous symptoms such as itching and burning. 1,23,24 
Table I Topical and systemic treatments of PRP

Topical treatments
Corticosteroids $\mathrm{s}^{23,25}$
Calcipotrio ${ }^{25}$
Tazarotene ${ }^{18,26}$
Emollients ${ }^{23,24}$ (urea $5-10 \%$, salycilic acid I-5\%)
Systemic treatments
First-line treatments
Isotretinoin ${ }^{27-34}$
Acitretin ${ }^{27-34}$
Second-line treatments
Methotrexate ${ }^{27,30,34}$
Acitretin + narrowband-UVB ${ }^{43}$
Acitretin + UVAI ${ }^{44}$
Acitretin + PUVA ${ }^{28}$
Cyclosporin ${ }^{48-50}$
Refractory forms
Anti-TNF-alpha inhibitors ${ }^{55-64}$ (infliximab, etanercept, and
adalimumab)
IL-23 and IL-I7 inhibitors ${ }^{54,65-71}$ (ustekinumab and sekukinumab
PDE4 inhibitor apremilast ${ }^{72}$

Abbreviations: PDE4, phosphodiesterase 4; PRP, pityriasis rubra pilaris; PUVA, psoralen-ultraviolet A; UVAI, ultraviolet AI.

In localized forms of PRP, topical therapy is the main therapeutic choice, ${ }^{23,24}$ including medium to high potency corticosteroids, keratolytics, emollients, and Vitamin D derived such as calcipotriol, ${ }^{25}$ tretinoin, and tazarotene. ${ }^{18,26}$

Topical treatments in association with systemic therapy are helpful to treat hyperkeratosis and palmoplantar keratoderma and to improve skin texture, reducing scaling, erythema, and induration, particularly, keratolitics $(5-10 \%$ urea or $5 \%$ salicylic acid) and emollients. ${ }^{27}$

\section{Systemic treatments}

Systemic treatments are indicated in moderate-to-severe disease. Traditional systemic treatment consists mainly in retinoids, which are actually considered as first-line therapy both in adult and in juvenile forms. ${ }^{1,27}$ Unfortunately, refractory cases that do not respond or relapse after drug interruption do exist. In these instances, treatment is challenging and a standard therapeutic protocol does not yet exist. Treatment is mainly based on case reports or case series of clinical experience because no controlled randomized trials have ever been performed because of the rarity of the condition. ${ }^{27}$ Nevertheless, in recent years, numerous case reports have demonstrated the efficacy of new therapeutic agents such as biological agents, in particular for refractory forms of PRP.

\section{Retinoids}

Systemic retinoids are considered as first-line systemic therapy for adults and children with PRP because most of the case reports and case series demonstrate their efficacy. ${ }^{27-39}$
Clinical response to retinoids, usually is evident after 3-6 months of therapy, but in some patients, longer treatment is needed. Isotretinoin and acitretin are the most used, but there are some data also about the efficacy of alitretinoin for PRP. ${ }^{27-30,35-38}$ In a retrospective study by Eastham et al, ${ }^{27}$ all the 12 patients treated with acitretin, eight patients receiving acitretin in monotherapy at dosage of $25-50 \mathrm{mg}$ /day, two patients receiving acitretin in association with methotrexate, and two patients receiving acitretin in combination with cyclosporine, reported partial or significant improvement during treatment. In a prospective study, isotretinoin $(2 \mathrm{mg} /$ $\mathrm{kg}$ /day) was used in 45 patients with PRP and, after 4 weeks of therapy, 28 (62\%) patients showed significant improvement. ${ }^{29}$ In another retrospective study including 15 patients treated with isotretinoin (primarily $40 \mathrm{mg}$ twice daily), 10 (67\%) patients had a complete clearance within an average of 25 weeks (range 16-44 weeks) and only three patients resulted refractory to treatment. ${ }^{30}$

In clinical practice, the recommended dosage of isotretinoin for adults with PRP is $1 \mathrm{mg} / \mathrm{kg} /$ day, while acitretin is used at the dosage of $0.5-0.75 \mathrm{mg} / \mathrm{kg} /$ day. However, high dosage of retinoids is not well tolerated because of frequent mucocutaneous side effects.

Most frequent adverse effects of retinoids are dry skin and mucous membranes, hyperlipidemia, transaminase elevations, and visual or bone changes. In children, retinoids could induce hyperostosis and premature epiphyseal closure especially in prepuberal patients treated with high doses. In addition, retinoids should be avoided in women of childbearing potential because of their teratogenic effect: pregnancy must be avoided during and for 3 years after treatment with acitretin and during and for 6 weeks after isotretinoin therapy.

Due to the rarity of the disease and the lack of standardized studies, there are no consistent data about treatment duration, risk of relapse at treatment discontinuation, or need for maintenance treatment.

\section{Phototherapy}

Ultraviolet B (UVB) phototherapy and psoralen-ultraviolet A (PUVA) therapy have been proved to be a successful treatment in some patients affected by PRP, ${ }^{39,40}$ although response to light is rather variable. Quite the opposite, exacerbations of PRP have been described in some patients. ${ }^{8,41,42}$ In consideration of reports of photoaggravated PRP, phototesting should be always performed before treatment. A valid alternative treatment in refractory forms to retinoids could be the combination of retinoids and phototherapy. Acitretin has been reported as a combined therapy with narrowband $\mathrm{UVB},{ }^{43}$ 
ultraviolet A1 (UVA1), ${ }^{44}$ and PUVA. ${ }^{28}$ In a recent case report of childhood PRP, phototherapy with narrowband-UVB has been proved to be successful after 2 months of treatment in combination with topical emollients, hydrocortisone cream, and calcipotriene, reaching $>90 \%$ cutaneous clearance. ${ }^{45}$

\section{Methotrexate}

Methotrexate is an alternative therapeutic option for PRP, as second-line treatment. ${ }^{27,34}$ It has been observed in retrospective studies the efficacy of weekly doses of 5-25 mg. Another retrospective study has demonstrated a favorable response in 8/14 patients with type I PRP: the average duration of treatment was 6 months (from 1 to 12 months). ${ }^{30}$ Another retrospective study has demonstrated the efficacy of methotrexate in association with retinoid, despite the increased risk for hepatotoxicity. ${ }^{33}$ In another study including five patients, refractory to retinoids methotrexate has been proved to be effective. ${ }^{34}$ Gastrointestinal distress is a frequent adverse event reported. Additional serious adverse effects that should be always checked are pancytopenia, hepatotoxicity, and pneumonitis. Moreover, it should be avoided in women of childbearing potential because of its theratogenic effect.

\section{Other immunosuppresants}

Other systemic agents such as azathioprine ${ }^{46,47}$ and cyclosporin ${ }^{48-50}$ have been reported as effective in the treatment of PRP in single case report, especially in refractory forms. In particular, cyclosporin could be considered a valid therapy for patients who do not respond to retinoids or methotrexate and could be used both in adult and juvenile forms. Also, fumaric acid esters may be an alternative option. ${ }^{51}$

\section{Biological agents}

In consideration of clinical and histological similarities between psoriasis and PRP, all the biological agents approved for psoriasis have also been used in the treatment of refractory forms of PRP or in patient resistant or ineligible to conventional systemic treatments. ${ }^{27,34}$ Being the pathogenesis of PRP still not clear, TNF-alpha has been hypothesized to be a key cytokine. ${ }^{52}$ More recently, inhibitors of the IL-23/IL-17 pathway have also been administrated successfully in PRP and increased levels of IL-17 have been found in PRP patients, providing a rationale for these new therapies..$^{53}$ However, clinical experiences about biological agents in the treatment of PRP is limited to case reports and case series and multicenter randomized clinical trials have not been performed because of low incidence of PRP. In contrast, cases of PRP refractory to biological agents are also observed, although rarely reported as recently described in a case of type IV PRP resistant to anti-TNF-alpha and IL-23 inhibitors. ${ }^{54}$

\section{Anti-TNF-alpha (infliximab, etanercept, and adalimumab)}

In the last 10 years, retrospective studies and case reports have shown that anti-TNF-alpha agents are effective in the treatment of PRP both in adult and in juvenile forms. ${ }^{53-64}$ A systematic review of type I PRP, including 15 patients treated with infliximab, etanercept, or adalimumab, have shown a complete response in 12/15 patients and a partial response in $2 / 15$ patients. Just in one case, no improvement has been observed. Six patients received anti-TNF-alpha as monotherapy, whereas nine patients were treated in association with acitretin or methotrexate..$^{63}$ In the majority of cases, TNF-alpha inhibitors have been used as second-line treatment in refractory PRP to traditional systemic treatment. In a case series of seven patients with refractory adult onset, PRP has proved the efficacy of infliximab (three patients) and etanercept (five patients), 5/7 patients received combination therapy with low-dose acitretin $(0.2 \mathrm{mg} / \mathrm{kg} /$ day $)$. All the patients experienced significant clearance at week 12 , and infliximab was associated with a more rapid response than etanercept. During follow-up, only a patient affected by type II PRP developed recurrence after 2 months from discontinuation. ${ }^{51}$ In a recent retrospective study of 40 patients with PRP performed in a third-level center, nine patients have been treated with anti-TNF-alpha agents with a favorable response within 5-7 weeks. Despite this, most of them received initial association therapy with another systemic drug (methotrexate, acitretin, or prednisone) ${ }^{27}$ In other case reports of refractory PRP treated with adalimumab, rapid and sustained remission was observed after only 4 weeks: no relapses were reported after treatment discontinuation. ${ }^{60-62}$ In patient affected by PRP type I treated with adalimumab and achieving clinical remission after 4 months, increased levels of mRNA of TNF-alpha were found in the lesional and perislesional normal skin. This finding is consistent with the observed clinical remission and supports the use of anti-TNF-alpha for the treatment of PRP. ${ }^{64}$

\section{IL-23 and IL-I 7 inhibitors (ustekinumab and secukinumab)}

Several case reports have described the use of ustekinumab in type I PRP, refractory to conventional systemic therapies, or anti-TNF-alpha agents. ${ }^{65-69}$ Ustekinumab demonstrated striking and rapid efficacy in reducing the signs and symptoms of the disease and also a long-term control of the disease. ${ }^{69} \mathrm{In}$ 
a recent cases series, lesional skin biopsy sample was taken from three patients affected by refractory PRP and mRNA expression of proinflammatory innate and T-cell-derived cytokines were measured. Gene expression analysis revealed an increase in T-helper (Th) 1 cytokines and in particular Th17 cytokines, such as IL-17A, IL-22, and IL-23. In one patient, levels of IL-17A from lesional skin samples were measured before and after treatment with ustekinumab and reduced after cutaneous improvement. This case report gives evidence of the role of the IL-23/Th17 axis in PRP, providing a rationale for targeting this pathway as a treatment option for refractory PRP. ${ }^{53}$ Secukinumab has been recently showed to be effective to treat PRP in two case reports. ${ }^{70,71}$ In the first one, it has been used in a patient with type I PRP, refractory to acitretin. ${ }^{70}$ In the second case, it has been proposed as a valid option to treat refractory type II PRP, with improvement of erythema and palmoplantar keratoderma observed after 2 weeks of treatment and no recurrence after a 6-month follow-up. ${ }^{71}$

\section{Other treatments}

A recent case has proved the efficacy of apremilast in the treatment of refractory PRP: after 4 weeks of therapy, the patient reported significant improvement and, at 6 months of follow-up, he/she showed complete clearance of skin lesions. ${ }^{72}$ Extracorporeal photochemotherapy was also used for the treatment of two patients with erythrodermic PRP type I in combination with systemic retinoids and cyclosporine, with good results. ${ }^{73}$ In another report, a case of type II adult-onset PRP was successfully treated with intravenous immunoglobulin. ${ }^{74}$

\section{Conclusion}

PRP is a rare inflammatory skin disease that can have a severe impact on patients' quality of life, especially in the chronic and refractory forms. Many therapeutic options have been experienced, but we do not have a standard protocol to treat PRP. Treatment of refractory forms remains challenging. Biological agents seem to represent a novel effective treatment for PRP, although multicenter randomized clinical trials should be performed before considering them as first-line option. Further insights into the pathogenesis of the disease will possibly disclose some clinical and phenotypic features in the different forms of PRP as predictive factors allowing a personalized therapeutic choice.

\section{Disclosure}

The authors report no conflicts of interest in this work.

\section{References}

1. Klein A, Landthaler M, Karrer S. Pityriasis rubra pilaris: a review of diagnosis and treatment. Am J Clin Dermatol. 2010;11(3):157-170.

2. Yang CC, Shih IH, Lin WL, et al. Juvenile pityriasis rubra pilaris: report of 28 cases in Taiwan. J Am Acad Dermatol. 2008;59(6):943-948.

3. Möhrenschlager M, Abeck D. Further clinical evidence for involvement of bacterial superantigens in juvenile pityriasis rubra pilaris (PRP): report of two new cases. Pediatr Dermatol. 2002;19(6):569.

4. Howe K, Foresman P, Griffin T, Johnson W. Pityriasis rubra pilaris with acantholysis. J Cutan Pathol. 1996;23(3):270-274.

5. Fuchs-Telem D, Sarig O, van Steensel MA, et al. Familial pityriasis rubra pilaris is caused by mutations in CARD14. Am J Hum Genet. 2012;91(1):163-170.

6. Takeichi T, Sugiura K, Nomura T, et al. Pityriasis rubra pilaris type $\mathrm{V}$ as an autoinflammatory disease by CARD14 mutations. JAMA Dermatol. 2017;153(1):66-70.

7. White KL. Pityriasis rubra pilaris. Dermatol Online J. 2003;9(4):6.

8. Evangelou G, Murdoch SR, Palamaras I, Rhodes LE. Photoaggravated pityriasis rubra pilaris. Photodermatol Photoimmunol Photomed. 2005;21(5):272-274.

9. Di Stefani A, Orlandi A, Chimenti S, Bianchi L. Phrynoderma: a cutaneous sign of an inadequate diet. Can Med Ass J. 2007;177(8):4-5.

10. Arnold AW, Buechner SA. Circumscribed juvenile pityriasis rubra pilaris. J Eur Acad Dermatol Venereol. 2004;18(6):705-707.

11. Martinez Calixto LE, Suresh L, Matsumura E, Aguirre A, Radfar L. Oral pityriasis rubra pilaris. Oral Sur Oral Med Oral Pathol Oral Radiol Endod. 2006;101(5):604-607.

12. Sehgal VN, Srivastav G. (Juvenile) pityriasis rubra pilaris. Int J Dermatol. 2006;45(4):438-446.

13. Griffiths A. Pityriasis rubra pilaris. Etiologic considerations. J Am Acad Dermatol. 1984;10(6):1086-1088.

14. Griffiths WA. Pityriasis rubra pilaris. Clin Exp Dermatol. 1980;5(1): 105-112.

15. Griffiths WA. Pityriasis rubra pilaris: the problem of its classification. J Am Acad Dermatol. 1992;26(1):140-142.

16. Miralles ES, Núñez M, De Las Heras ME, Pérez B, Moreno R, Ledo A. Pityriasis rubra pilaris and human immunodeficiency virus infection. Br J Dermatol. 1995;133(6):990-993.

17. Bragg J, Witkiewicz A, Orlow SJ, Schaffer JV. Pityriasis rubra pilaris, type IV. Dermatol Online J. 2005;11(4):14.

18. Caldarola G, Zampetti A, De Simone C, et al. Circumscribed pityriasis rubra pilaris type IV. Clin Exp Dermatol. 2007;32(4):471-472.

19. Zalaudek I, Argenziano G, Di Stefani A, et al. Dermoscopy in general dermatology. Dermatology. 2006;212(1):7-18.

20. Lallas A, Apalla Z, Karteridou A, Lefaki I. Photoletter to the editor: dermoscopy for discriminating between pityriasis rubra pilaris and psoriasis. J Dermatol Case Rep. 2013;7(1):20-22.

21. Pietroleonardo L, Di Stefani A, Campione E, et al. Confocal reflectance microscopy in pityriasis rubra pilaris. $J$ Am Acad Dermatol. 2013;68(4):689-691.

22. Soeprono FF. Histologic criteria for the diagnosis of pityriasis rubra pilaris. Am J Dermatopathol. 1986;8(4):277-283.

23. Allison DS, El-Azhary RA, Calobrisi SD, Dicken CH. Pityriasis rubra pilaris in children. J Am Acad Dermatol. 2002;47(3):386-389.

24. Selvaag E, Haedersdal M, Thomsen K. Pityriasis rubra pilaris: a retrospective study of 12 patients. J Eur Acad Dermatol Venereol. 2000;14(6):514-515.

25. Van de Kerkhof PC, Steijlen PM. Topical treatment of pityriasis rubra pilaris with calcipotriol. Br J Dermatol. 1994;130(5):675-678.

26. Karimian-Teherani D, Parissa M, Tanew A. Response of juvenile circumscribed pityriasis rubra pilaris to topical tazarotene treatment. Pediatr Dermatol. 2008;25(1):125-126.

27. Eastham AB, Femia AN, Qureshi A, Vleugels RA. Treatment options for pityriasis rubra pilaris including biologic agents: a retrospective analysis from an academic medical center. JAMA Dermatol. 2014;150(1) 92-94. 
28. Gemmeke A, Schönlebe J, Koch A, Wollina U. Pityriasis rubra pilaris - a retrospective single center analysis over eight years. J Dtsch Dermatol Ges. 2010;8(6):439-444.

29. Goldsmith LA, Weinrich AE, Shupack J. Pityriasis rubra pilaris response to 13-cis-retinoic acid (isotretinoin). J Am Acad Dermatol. 1982;6(4 pt 2 suppl):710-715.

30. Dicken $\mathrm{CH}$. Treatment of classic pityriasis rubra pilaris. J Am Acad Dermatol. 1994;31(6):997-999.

31. Borok M, Lowe NJ. Pityriasis rubra pilaris. Further observations of systemic retinoid therapy. J Am Acad Dermatol. 1990;22(5 pt 1):792-795.

32. Clayton BD, Jorizzo JL, Hitchcock MG, et al. Adult pityriasis rubra pilaris: a 10-year case series. J Am Acad Dermatol. 1997;36(6 pt 1):959-964.

33. Cohen PR, Prystowsky JH. Pityriasis rubra pilaris: a review of diagnosis and treatment. J Am Acad Dermatol. 1989;20(5 pt 1):801-807.

34. Ross NA, Chung HJ, Li Q, Andrews JP, Keller MS, Uitto J. Epidemiologic, clinicopathologic, diagnostic, and management challenges of pityriasis rubra pilaris: a case series of 100 patients. JAMA Dermatol. 2016;152(6):670-675.

35. Molin S, Ruzicka T. Treatment of refractory pityriasis rubra pilaris with oral alitretinoin: case report. Br J Dermatol. 2010;163(1):221-223.

36. Schmitt L, Inhoff O, Dippel E. Oral alitretinoin for the treatment of recalcitrant pityriasis rubra pilaris. Case Rep Dermatol. 2011;3(1):85-88.

37. Amann PM, Susic M, Glüder F, Berger H, Krapf W, Löffler H. Alitretinoin (9-cis retinoic acid) is effective against pityriasis rubra pilaris: a retrospective clinical study. Acta Derm Venereol. 2015;95(3):329-331.

38. Pampín A, Gómez-de la Fuente E, Caro Gutiérrez MD, López-Estebaranz JL. Successful treatment of atypical adult pityriasis rubra pilaris with oral alitretinoin. J Am Acad Dermatol. 2013;69(2):e105-e106.

39. Mainardi L, Caccialanza M, Piccinno R. Efficacy of photochemotherapy in a case of pityriasis rubra pilaris. G Ital Dermatol Venereol. 1990;125(11):537-538.

40. Kaskel P, Grundmann-Kollmann M, Schiller PI, et al. Bath-PUVA as a treatment for Pityriasis rubra pilaris provoked by ultraviolet B. $\mathrm{Br} J$ Dermatol. 1999;140(4):769-770.

41. Yaniv R, Barzilai A, Trau H. Pityriasis rubra pilaris exacerbated by ultraviolet B phototherapy. Dermatology. 1994;189(3):313.

42. Marguery MC, Durand-Malgouyres C, Bayle-Lebey P, Dupin P, Bazex J. Photosensitive and phototriggered pityriasis rubra pilaris. Photodermatol Photoimmunol Photomed. 1994;10(1):42-45.

43. Kirby B, Watson R. Pityriasis rubra pilaris treated with acitretin and narrow-band ultraviolet B (Re-TL-01). Br J Dermatol. 2000;142(2):376-377.

44. Herbst RA, Vogelbruch M, Ehnis A, et al. Combined ultraviolet A1 radiation and acitretin therapy as a treatment option for pityriasis rubra pilaris. Br J Dermatol. 2000;142(3):574-575.

45. Massa AF, Vasconcelos P, Soares de Almeida L, Filipe P. Pityriasis rubra pilaris mixed type III/IV successfully treated with narrow bandultraviolet B. Indian J Dermatol Venereol Leprol. 2015;81(4):435.

46. Hunter GA, Forbes IJ. Treatment of pityriasis rubra pilaris with azathioprine. Br J Dermatol. 1972;87(1):42-45.

47. Schram ME, Borgonjen RJ, Bik CM, et al. Off-label use of azathioprine in dermatology: a systematic review. Arch Dermatol. 2011;147(4):474 488.

48. Wetzig T, Sticherling M. Juvenile pityriasis rubra pilaris: successful treatment with ciclosporin. Br J Dermatol. 2003;149(1):202-203.

49. Usuki K, Sekiyama M, Shimada T, Shimada S, Kanzaki T. Three cases of pityriasis rubra pilaris successfully treated with cyclosporin A. Dermatology. 2000;200(4):324-327.

50. Rosenbach A, Lowe NJ. Pityriasis rubra pilaris and cyclosporine. Arch Dermatol. 1993;129(10):1346-1348.

51. Coras B, Vogt TH, Ulrich H, et al. Fumaric acid esters therapy: a new treatment modality in pityriasis rubra pilaris? $\mathrm{Br} J$ Dermatol. 2005;152(2):388-389.

52. Garcovich S, Di Giampetruzzi AR, Antonelli G, Garcovich A, Didona B. Treatment of refractory adult-onset pityriasis rubra pilaris with TNF-alpha antagonists: a case series. J Eur Acad Dermatol Venereol. 2010;24(8):881-884.
53. Feldmeyer L, Mylonas A, Demaria O, et al. Interleukin 23-helper T cell 17 axis as a treatment target for pityriasis rubra pilaris. JAMA Dermatol. 2017;153(4):304-308.

54. Di Lernia V, Ficarelli E, Zanelli M. Ineffectiveness of tumor necrosis factor- $\alpha$ blockers and ustekinumab in a case of type IV pityriasis rubra pilaris. Indian Dermatol Online J. 2015;6(3):207-209.

55. Davis KF, Wu JJ, Murase JE, et al. Clinical improvement of pityriasis rubra pilaris with combination etanercept and acitretin therapy. Arch Dermatol. 2007;143(12):1597-1599.

56. Seckin D, Tula E, Ergun T. Successful use of etanercept in type I pityriasis rubra pilaris. Br J Dermatol. 2008;158(3):642-644.

57. Cox V, Lesesky EB, Garcia BD, O’Grady TC. Treatment of juvenile pityriasis rubra pilaris with etanercept. J Am Acad Dermatol. 2008;59(5 suppl):S113-S114.

58. Manoharan S, White S, Gumparthy K. Successful treatment of type I adult-onset pityriasis rubra pilaris with infliximab. Australas J Dermatol. 2006;47(2):124-129.

59. Ruiz-Genao DP, Lopez-Estebaranz JL, Naz-Villalba E, et al. Pityriasis rubra pilaris successfully treated with infliximab. Acta Derm Venereol. 2007;87(6):552-553.

60. Walling HW, Swick BL. Pityriasis rubra pilaris responding rapidly to adalimumab. Arch Dermatol. 2009;145(1):99-101.

61. Schreml S, Zeller V, Babilas P, et al. Pityriasis rubra pilaris successfully treated with adalimumab. Clin Exp Dermatol. 2010;35(7):792-793.

62. O'Kane D, Devereux CE, Walsh MY, Hoey SE. Rapid and sustained remission of pityriasis rubra pilaris with adalimumab treatment. Clin Exp Dermatol. 2010;35(4):155-156.

63. Petrof G, Almaani N, Archer CB, Griffiths WA, Smith CH. A systematic review of the literature on the treatment of pityriasis rubra pilaris type 1 with TNF-antagonists. J Eur Acad Dermatol Venereol. 2013;27(1):131-135.

64. Zhang YH, Zhou Y, Ball N, Su MW, Xu JH, Zheng ZZ. Type I pityriasis rubra pilaris: upregulation of tumor necrosis factor alpha and response to adalimumab therapy. J Cutan Med Surg. 2010;14(4):185-188.

65. Wohlrab J, Kreft B. Treatment of pityriasis rubra pilaris with ustekinumab. Br J Dermatol. 2010;163(3):655-656.

66. Ruiz Villaverde R, Sánchez Cano D. Successful treatment of type 1 pityriasis rubra pilaris with ustekinumab therapy. Eur J Dermatol. 2010;20(5):630-631.

67. Eytan O, Sarig O, Sprecher E, van Steensel MA. Clinical response to ustekinumab in familial pityriasis rubra pilaris caused by a novel mutation in CARD14. Br J Dermatol. 2014;171(2):420-422.

68. Balestri R, Bardazzi F, Antonucci A. Should ustekinumab really be used as first-line biological therapy in pityriasis rubra pilaris? Br J Dermatol. 2010;163(4):896-897.

69. Di Stefani A, Galluzzo M, Talamonti M, Chiricozzi A, Costanzo A, Chimenti S. Long-term ustekinumab treatment for refractory type I pityriasis rubra pilaris. J Dermatol Case Rep. 2013;7(1):5-9.

70. Schuster D, Pfister-Wartha A, Bruckner-Tuderman L, Schempp CM. Successful treatment of refractory pityriasis rubra pilaris with secukinumab. JAMA Dermatol. 2016;152(11):1278-1280.

71. Gauci ML, Jachiet M, Gottlieb J, et al. Successful treatment of type II pityriasis rubra pilaris with secukinumab. JAAD Case Rep. 2016;2(6): $462-464$.

72. Krase IZ, Cavanaugh K, Curiel-Lewandrowski C. Treatment of refractory pityriasis rubra pilaris with novel phosphodiesterase 4 (PDE4) inhibitor apremilast. JAMA Dermatol. 2016;152(3):348-350.

73. Hofer A, Müllegger R, Kerl H, Wolf P. Extracorporeal photochemotherapy for the treatment of erythrodermic pityriasis rubra pilaris. Arch Dermatol. 1999;135(4):475-476.

74. Kerr AC, Ferguson J. Type II adult-onset pityriasis rubra pilaris successfully treated with intravenous immunoglobulin. $\mathrm{Br} J$ Dermatol. 2007;156(5):1055-1056. 


\section{Publish your work in this journal}

Clinical, Cosmetic and Investigational Dermatology is an international, peer-reviewed, open access, online journal that focuses on the latest clinical and experimental research in all aspects of skin disease and cosmetic interventions. This journal is included on PubMed. The manuscript management system is completely online and includes a very quick and fair peer-review system, which is all easy to use. Visit http://www.dovepress.com/testimonials.php to read real quotes from published authors

Submit your manuscript here: https://www.dovepress.com/clinical-cosmetic-and-investigational-dermatology-journal 